\title{
Diapause des Cyclopides d'un écosystème lacustre peu profond (Lac de Créteil, France).
}

\author{
Gérard Lacroix 1 \\ Françoise Lescher-Moutoué1
}

Mots-clés : Cyplopidae, lac, cycles de vie, diapause, facteurs immédiats, signification adaptative.

La diapause des Cyclopides pélagiques d'un lac tempé ré peu profond (Créteil, France) a été étudiée sur un cycle annuel. Une dormance estivale affecte les copépodites IV de Cyclops vicinus (avril-mai à février) et de Diacyclops bicuspidatus (mars à novembre); une diapause hivernale est observée chez les copépodites IV de Thermocyclops crassus et chez les subadultes de Thermocyclops oithonoides (septembre à avril).

Quelques subadultes de Th. oithonoides ont un arrèt de développement sans pénétration dans le sédiment (diapause active).

La biomasse moyenne de la fraction diapausante égale $36 \%(15 \%$ à $80 \%)$ de la biomasse totale des Cyclopides pendant la periode d'étude. Les effectifs benthiques, compris entre $27000 \mathrm{ind} . \mathrm{m}^{-2}$ et $350000 \mathrm{ind} . \mathrm{m}^{-2}$, representent un potent iel de recolonisation du domaine pélagique de 6 ind. $1^{-1}$ à 78 ind. $1^{-1}$.

Alors que la diapause hivernale de Th. oithonoides et de Th. crassus est probablement une adaptation à des conditions climatiques défavorables, la diapause estivale de $C$. vicinus serait une stratégie a ": ptative d'évitement de la prédation.

Diapause in Cyclopoids of a limnetic shallow ecosystem (Lake of Crétell, France).

Keywords: Cyclopidae, lake, life histories, diapause, proximate factors, adaptive significance.

The diapause of pelagic Cyclopoids was studied in a temperate shallow lake (Créteil, France) over a period of one year. A surmmer dormancy occurred in copepodid stage IV of Cyclops vicinus (from Aprit-May to February) and Diacyclops bicuspidatus (from March to November). Copepodid stage IV of Thermocyclops crassus and subadults of Thermocyclops oithonoides overwintered in the sediment from September to April.

A few subadults of Th. oithonoides had an arrested development without penetration in the mud (active diapause).

The mean biomass of resting stages was cqual to $36 \%(15 \% .80 \%)$ of the total Cyclopoid biomass. The benthic abun. dance varied from 27000 ind. $\mathrm{m}^{-2}$ to 350000 ind. $\mathrm{m}^{-2}$ and gave a potential recolonization level of the open water area of 6.78 ind. $1^{-1}$

Whereas the winter diapause of Th. oithonoides and Th. crassus is very likely to be an adaptation to unfavorable climatic conditions, the summer diapause of $C$. vicinas could be a strategy of escape from predation pressure.

1. Ecole Normale Supérieure, Laboratoire de Zoologie, 46, rue: d'Ulm. 75230 Paris Cedex 05, France.

1. L'espèce nord-américaine Diacyclops thomasi (Forbes) à propos de laquelle Birge el Juday (1908) ont observé pour la première fois l'existence d'une diapause au stade copépodite chex. les Cycle. pidae, est considerée souvent comme synonyme de $D$. bictuspida. itus (Claus) ou comrse une de ses sous-espèces. En accord avec Kiefer (1978 a), nous considérons qu'it s'agit de deux espèces dist inctes.
Une diapause a été observée dans le cycle de vie de nombreux Cyclopides par divers auteurs (cf. Elgmork 1980). Elle se produit en général à un stade copépodite précis; plus rarement, elle affecte plusieurs stades juvéniles d'une mème espèce (Elgmork 1962). Physiologiquement équivalente chez les Insec. tes et les Cyclopides (Cole 1953, Elgmork et Nilssen 
1978), cette diapause semble essentiellement contrôlée par la photopériode et lat température (Einsle 1964, Spindler 1971, Watson et Smallman $1971 a$, Sarvala 1979).

La signification adaptative de la diapause des Cyclopides n'a pas toujours été clairement démontrée. Si pour certaines espèces les facteurs physiques climatiques suffisent à l'expliquer, pour d'autres il apparaît maintenant qu'elle doit être considérée comme une stratégie de refuge face à la prédation, ou encore d'optinisation de la reproduction (Nilssen 1978, 1980, Elgmork 1980).

Le recensement des individus diapausants dans le sédiment est donc indispensable à une bonne compréhension du cycle de vie des Cyclopides. Ainsi, dans le lac de Créteil, le suivi de l'évolution simultanée des écophases benthique et pélagique des Cyclopides a permis de déterminer les conditions spatio-temporelles de la diapause de Cyclops vicinus vicinus (Ulianine), Diacyclops bicuspidatus (Claus), Thermocyclops oithonoides (Sars) et Th. crassus (Fischer). Seul Acanthocyclops robustus (Sars) n'est pas entré en diapause au cours de la période d'observation, alors qu'il peut avoir une latence hivernale dans d'autres lacs européens. La comparaison de nos résultats avec ceux obtenus par divers auteurs en d'autres sites nous a conduits à effectuer une analyse critique des facteurs immédiats qui régissent l'induction et la levée de la diapause des Cyclopides de Créteil, puis à en discuter la valeur adaptative.

\section{1. - Description du milieu}

Le lac de Créteil occupe l'emplacement d'une ancienne sablière située en zone périurbaine à $20 \mathrm{~km}$ de Paris entre la Seine et la Marne. Ce plan d'eau aux rives abruptes a un fond relativement plat. Milieu fermé, il est alimenté par la nappe phréatique et, secondairement, par les eaux de pluie; le temps de renouvellement est de 215 jours.

Les principales caractéristiques du lac sont regroupées dans le tableau 1 . L'installation d'un gradient thermique en avril-mai entraine une stratification estivale de l'oxygène (fig. 1), le déficit au niveau du fond pouvant atteindre l'anoxie. L'action du vent provoque périodiquement une homogénéisation thermique et une réoxygénation de la colonne d'eau.

Tableau 1. Caractéristiques physicu-chimiques et des peuplements planc. toniques et piscicole du Lac de Créteil (Testard 1983).

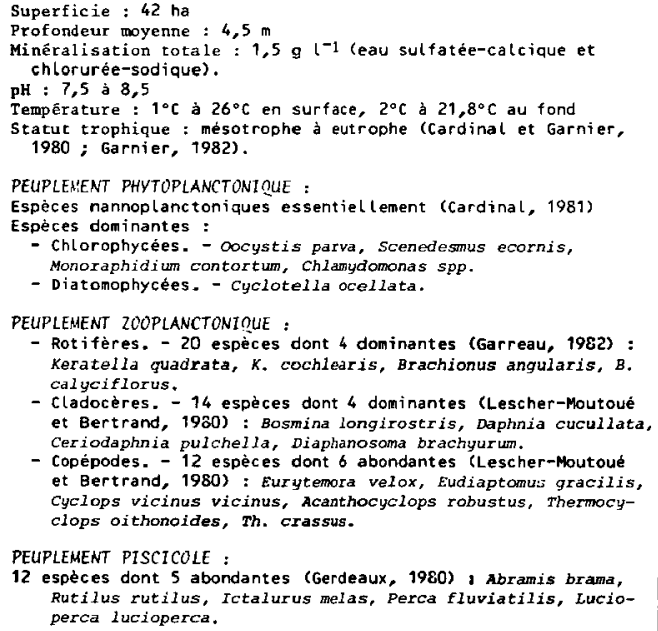



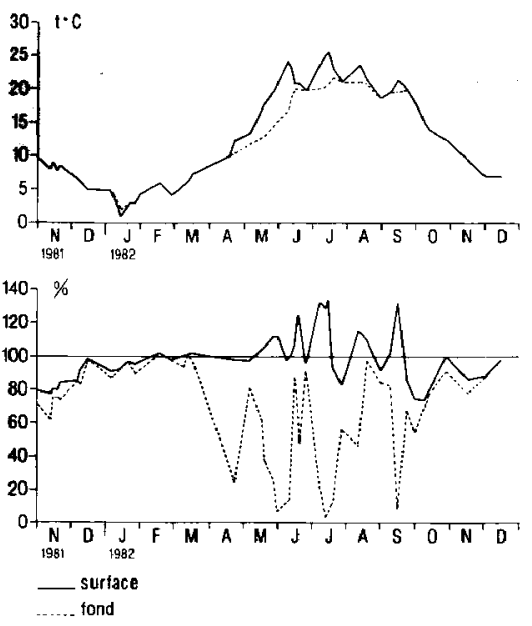

Fig. 1. Variations saisonnières de la température de l'eau et l'oxygène dissous (en pourcentage de la teneur de saturation)du Lac de Créteil.

\section{2. - Matériel et méthodes}

L'échantillonnage a été effectué dans la partie centrale du lac, selon un pas de temps bimensuel ou hebdomadaire. au cours d'un cycle annuel de novembre 1981 à décembre 1982. Afin de connaître l'abondance des populations dont étaient issus les individus diapausants, l'évolution du peuplement pélagique a été analysée à partir de janvier 1981 .

Pour le zcoplancton de la zone pélagique, on a utilisé une bouteille à prélèvement de deux litres de type Friedinger immergée à quatre niveaux $(0,5 \mathrm{~m} ; 1,5 \mathrm{~m} ; 2,5 \mathrm{~m}: 3.5 \mathrm{~m})$. L'èchantillon de huit litres ainsi obtenu a été filtré sur un filet de $25 \mu \mathrm{m}$, le filtrat étant immédiatement fixé au formol à $4 \%$.

La couche d'eau profonde immédiatement supérieure au substrat et le sédiment ont été prélevés avec un carottier en plexiglass de $50 \mathrm{~cm}^{2}$ de section (modèle adapté de Milbrink 1971).

La colonne d'eau retentue dans le carottier a une hauteur de $50 \mathrm{~cm}$ et un volume de 2,5 litres environ; elle est séparée du sédiment par siphonnage; le zooplancton retenu sur filtre de $25 \mu \mathrm{m}$ est fixé comme précédemment. L'analyse de la dispersion spatiale des individus présents dans cette couche d'eau profonde, par test de $\mathbf{X}^{2}$ sur dix échantillons prélevés simultanément, ne permet pas d'écar- ter l'hypothèse d'une distribution au hasard des Cyclopi. des à cette échelle d'observation. Par la suite, trois à huit relevés effectués à chaque date de prélèvement ont été réunis en un seul echantillon.

Dans chaque échantilton de la zone pélagique ou de la couche profonde, 100 à 150 Cyclopides étaient généralement décomptés par espece et par stade (copépodites I à $V$, mâles, femelles). Les échantillons de faible abondance ont été décomptés dans leur totalité.

Pour les sédiments, la fraction de granulométrie inférieure à $56 \mu \mathrm{m}$ a été éliminée par lavage dans un filet. Les Copépodes ont été fixés au formol à $4 \%$.

L'étude de la répartition verticale des Copépodes en diápause dans le sédiment a été effectuée en découpant les carottes congelées en tranches de un centimètre. La profondeur maximale d'enfouissement avoisinant $10 \mathrm{~cm}$, seuls les dix premiers centimètres ont été conservés dans notre étude (fig. 2). L'analyse de la dispersion horizontale dans le sédiment a été réalisée à partir de dix carottes préle. vées simultanément dans la mème station. A cet te échelle d'observation, la distribution est de caractère agrégatif. Trois à huit carot tes par échantillon ont pu être étudiées. La distribution contagieuse des Cyclopides en diapause ainsi que la petite taille des échantillons ont justifié l'emploi de la transformation logari thmique des données pour le calcul des intervalles de confiance de la moyenne au seuil de probabilité 0,95 (Elliott 1971).

\section{3. - Résultats}

\section{1. - Description des individus diapausants}

Les stades touchés par la diapause sont les copépodites IV de $C$. vicinus, de $D$. bicuspidatus et de $T h$. crassus, et les copépodites V de Th. oithonoides. Les individus diapausants contiennent généralement des gouttelettes lipidiques, bien visibles au moment de l'entrée en diapause, variables en nombre, en dimension et en couleur (incolore à rouge vif). Le tube digestif est le plus souvent vide. Les vacuoles à concrétions de l'estomac et les bouchons abdominaux décrits par Wierzbicka $(1966,1972)$ n'ont pas été vus avec certitude, seulement peut-être pour de rares $D$. bicuspidateis et $T h$. crassus. Les copépodites IV de $C$. vicinus sont fréquemment pliés à angle droit, leurs pattes natatoires étant rabattues vers l'avant. A leur sortie de diapause, la plupart des copépodites IV de $C$. vicinus et quelques-uns de $T h$. crassus avaient des " boucliers céphaliques" (Elgmork 1962, Wierzbicka 1966, Champeau 1970). Ces boucliers semblent constitués de fines particules agglomérées sur un mucus adhérant à la cuticule dé part et d'autre du céphalothorax. 


\section{2. - Répartition verticale dans le sédiment}

Les Cyclopides pénètrent activement dans le sédiment (Elgmork 1967). Leur profondeur d'enfouissement est en corrélation positive avec leur taille (fig. 2). Les copépodites IV de $C$. vicinus (longueur moyenne : $845 \mu \mathrm{m}$ ) peuvent s'enfoncer jusqu'à la profondeur de $10 \mathrm{~cm}$ dans le sédiment de Créteil, avec une densité maximale d'individus entre 3 et $4 \mathrm{~cm}$. Les copépodites IV de $T h$. crassus (longueur moyenne : $538 \mu \mathrm{m}$ ) sont surtout présents dans les deux premiers centimètres et n'ont pas été observés au-delà de $4 \mathrm{~cm}$. Quant aux copépodites IV de D. bicuspidatus (longueur moyenne : $774 \mu \mathrm{m}$ ), ils ont été récoltés jusqu'à $6 \mathrm{~cm}$ de profondeur. Ces résultats concordent avec ceux d'Elgmork et Nilssen (1978) pour quatre stades de développement de $C$. scutifer et avec les observations de Sarvala (1979) sur les genres Cyclops, Mesocyclops et Thermocyclops.

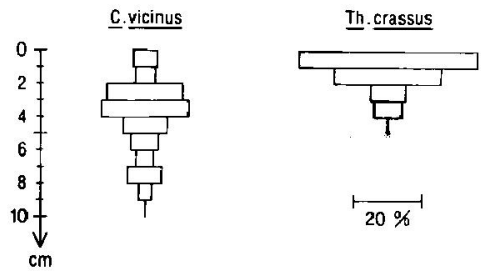

Fig. 2. Distribution verticale (en pourcentage) des copépodites IV de Cyclops vicinus et de Thermocyclops crassus en diapause dans le sédiment du Lac de Créteil.

\section{3. - Importance quantitative de la fraction ben- thique du peuplement}

La présence permanente de Copépodes diapausants dans les sédiments traduit la diversité des cycles biologiques des Cyclopides du lac de Créteil (fig. 4 à 8). La biomasse des individus diapausants constitue de $15 \%$ à $80 \%$ de la biomasse de l'ensemble des Cyclopides actifs et inactifs. Ce rapport est minimal en fin d'hiver et en fin d'été, maximal en fin d'automne et fin de printemps. La biomasse moyenne de la fraction benthique est de $444 \mathrm{mg}$ $\mathrm{m}^{-2}$ (poids sec) entre novembre 1981 et novembre 1982 ; elle représente ainsi $36 \%$ de la biomasse moyenne totale des Cyclopides. Le potentiel de recolonisation du peuplement pélagique est compris entre 6 ind. $\mathrm{I}^{-1}$ et 78 ind. $\mathrm{I}^{-1}$.

\section{4. - La diapause dans le cycle de vie}

La figure 3 représente l'évolution saisonnière de l'écophase pélagique des Cyclopides du lac de Créteil de janvier 1981 à décembre 1982. Seul, A. robustus est pérenne. C. vicinus est dominant dans le peuplement pendant la saison froide et sa diapause est estivale. $D$. bicuspidatus a lui aussi une diapause estivale mais l'évolution de sa population pélagique, d'importance négligeable, n'a pas pu être étudiée. Une diapause hivernale caractérise les cycles de $T h$. oithonoides et de $T h$. crassus; avec $A$. robustus, ces deux especes sont dominantes habituellement dans le peuplement copépodologique estival.

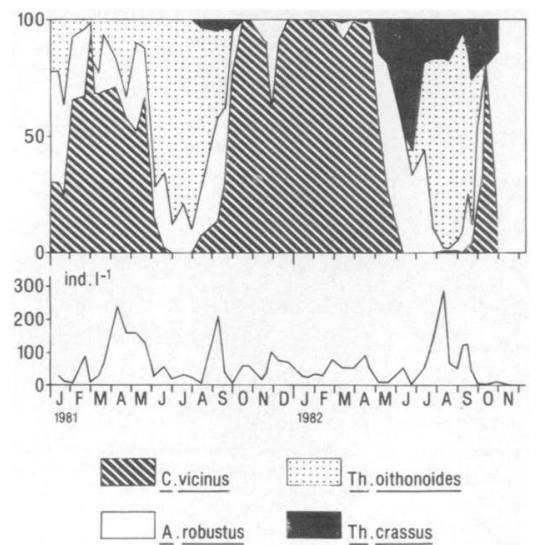

Fig. 3. Variations saisonnières (en pourcentage) des espèces représentatives du peuplement de Cyclopides du Lac de Créteil. le graphique inférieur représente l'évolution saisonnière des effectifs des stades copépodites et adultes de ce peuplement.

\subsection{1. - Cyclops vicinus vicinus}

C. vicinus est présent en phase active dans le lac de Créteil de la fin de l'été à la fin du printemps ; cinq générations se sont développées d'août 1981 à avril 1982. Il disparaît en général de la zone pélagique pendant une courte période (juin à aoûtseptembre).

L'entrée en diapause des copépodites IV commence fin avril-début mai pour une photopériode de 14 heures environ et une température de $13^{\circ} \mathrm{C}$ à $15^{\circ} \mathrm{C}$ (fig. 1 et 4 ). Elle est précédée par une migra- 
tion massive des copépodites IV vers le fond du lac : ainsi, le 29 avril 1982, leur densité à proximité du sédiment était 12,5 fois supérieure à leur densité en zone pélagique. Les variations des effectifs benthiques de la population lors de l'induction de la diapause en 1982 sont masquées par le maintien d'un grand nombre de copépodites IV dans le sédiment et par l'entrée en latence d'un nombre restreint d'in. dividus au printemps (fig. 4).

La réactivation débute fin août-début septembre (fig. 3 et 4). De septembre à février, la récolte régulière de copépodites IV pélagiques porteurs d'agglomérats céphalothoraciques prouve que la réactivation de la fin de l'èté n"affecte pas tous les individus diapausants et donc que la levée de la diapause s'étale dans le temps. Les dénombrements effectués à partir de novembre 1981 confirment ces observations (fig. 4) : 240500 ind. $m^{-2}$ restaient encore enfouis dans le sédiment en novembre-décembre 1981, ce qui correspond à un potentiel de recoloni-
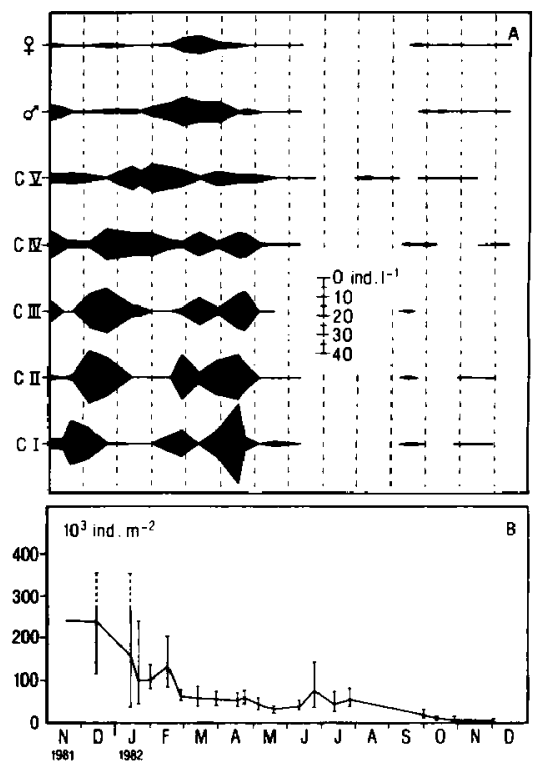

Fig. 4. Succession des stades copépodites et adultes pélagiques de Cyclops vicinus (A) et évolution comparé des copépodites IV en diapause dans le sédiment (B). sation de 50 ind. 1-1. C'est en janvier-février 1982 que s'est effectuée leur réactivation massive. Szlauer (1963) signale de même une réactivation partielle des C. vicinus diapausants du lac Stary Dwor (Pologne) au moment de la réoxygénation automnale, la fraction restante se maintenant dans le sédiment jusqu'à la réoxygénation printanière. George (1973) observe la levée de diapause de $C$. vicinus dans le réservoir d'Eglwys Nynydd (Pays de Galles) de février à mars. Enfin, Vijverberg (1977) observe cette émergence en septembre dans le réservoir de Tjeukemeer (PaysBas).

Il semble y avoir une dépendance entre l'importance des fractions benthique et pélagique de la population de $C$. vicinus à Créteil. On peut relier sans aucun doute la grande quantité d'individus dia. pausants en novembre 1981 à la présence d'un effecif important au moment de l'induction de la dia. pause ( 82 à 168 copépodites + adultes $1^{-1}$ d'avril à juin 1981, fig. 3) qui a pu assurer ainsi le passage à la vie latente d'un grand nombre d'individus. Ceci pourrait expliquer, en partie tout au moins, le développement inhabituel de l'automne 1981 (60 copé. podites + adultes $1-1$ ). En 1982 au contraire, la densité pélagique de $C$. vicinus était négligeable dès le debut du mois de mai, la diapause touchant de ce fait un nombre restreint de copépodites IV (fig. 4). Au moment de la levée de la diapause à la fin de l'été. cette faible fraction benthique n'aurait pas permis un demarrage satisfaisant de la population péla. gique dont les effectifs sont restes inférieurs à 4 copépodites + adultes $1^{-1}$ de septembre à décembre 1982 .

C. vicinus présente donc régulièrement à Créteil une diapause estivale. Il peut aussi, selon les années, maintenir simultanément une population pélagique plus ou moins abondante (Lescher-Moutoué \& Bertrand 1980). Ce maintien estival existe aussi dans les lacs de Constance (Einsle 1964) et d'Eglwys Nynydd (George 1973). Au contraire, la disparition estivale de la fraction pélagique est totale dans le réservoir de Tjeukemeer (Vijverberg 1977) et dans le lac Stary Dwor (Szlauer 1963).

\subsection{2. - Diacyclops bicuspidatus}

D. bicuspidatus ne s'est jamais reproduit de façon notable dans le lac de Créteil de 1978 à 1982 et a été observé seulement à proximité du sédiment aux épo- 
ques précédant et suivant immédiatement la phase de diapause. Les copépodites IV s'enfouissent dans le sédiment en mars (fig. 5). La photopériode est alors de $12 \mathrm{~h} 30 \mathrm{mn}$ environ et la température de l'eau est comprise entre $6^{\circ} \mathrm{C}$ et $8^{\circ} \mathrm{C}$ (fig. 1). La levée de diapause se produit en novembre. L'abondance moyenne des copépodites IV dans le sédiment est de 3930 ind $m^{-2}$ d'avril à octobre 1982, ce qui représente un potentiel de recolonisation de l'eau libre inférieur à 1 ind. $\mathrm{l}^{-1}$.

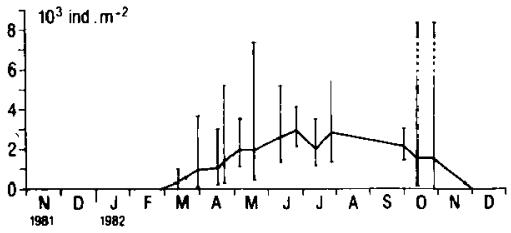

Fig. 5. Évolution saisonnière des densités de copépodites IV de Diacyclops bicuspidatus en diapause dans le sédiment.

Peu de travaux font état du cycle de vie en milieu pérenne de $D$. bicuspidatus; cette espece en effet colonise rarement les lacs. Einsle (1965) dans une étude sur la biologie de $D$. bicuspidatus vivant dans le lac de Constance, où il a été découvert assez récemment (Kiefer 1963), ne mentionne pas de phase de diapause. Aussi, à notre connaissance, la première observation d'une diapause estivale au stade IV en milieu lacustre pérenne est due à Vijverberg $(1977)^{1}$. En milieu temporaire, au contraire, $D$. bicuspidatzis présente régulièrement une période de repos (Roy 1932 in Champeau 1970; Champeau 1963, Wierzbicka 1966).

\subsection{3. - Thermocyclops oithonoides}

L'espèce thermophile Th. oithonoides atteint ses densités maximales à la fin de l'été dans le lac de Créteil. En saison froide, on observe encore quelques individus, principalement des subadultes (LescherMoutoué \& Bertrand 1980).

La réactivation des subadultes se situe fin mars pour des températures de l'eau voisines de $6^{\circ} \mathrm{C}$ à $8^{\circ} \mathrm{C}$; les mâles apparaissent dans le plancton pendant la première quinzaine d'avril; la quantité des femelles s'accroit ensuite sensiblement. Le nombre de générations est variable suivant les années ; ainsi, en 1982, deux générations ont été distinguées après le recensement de femelles ovigères au début de juillet. La population a atteint un maximum de $\mathbf{2 3 2}$ copépodites + adultes $1^{-1}$ le 11 août.

L'entrée en diapause des subadultes se produit vers la fin du mois de septembre $\left(5600\right.$ ind. $\mathrm{m}^{-2}$ au maximum) alors que quelques subadultes restent actifs dans le lac (fig. 6). La diapause des subadultes de Th. oithonoides dans le lac de Créteil est donc particulière: une partie d'entre eux s'enfouit normalement dans le sédiment, l'autre partie présente une phase d'arrêt de croissance (ou diapause active, selon Elgmork 1980). Dans les régions septentrionales, divers auteurs ont mentionné une diapause totale de Th. oithonoides (Elgmork 1958, 1973, Szlauer 1963, Sarvala 1979).

La fraction benthique très réduite et l'absence simultanée d'une fraction pélagique résiduelle de novembre 1981 à mai 1982, pourraient être une conséquence du fajble développement estival de $T h$. oithonoides: 77.5 copépodites + adultes $1^{-1}$ au maximum le 7 septembre 1981 ( $\mathrm{fg} .3$ et 6). Une telle interdépendance ent re les deux fractions de la population a été notée par ailleurs à propos de $C$. vicintus.

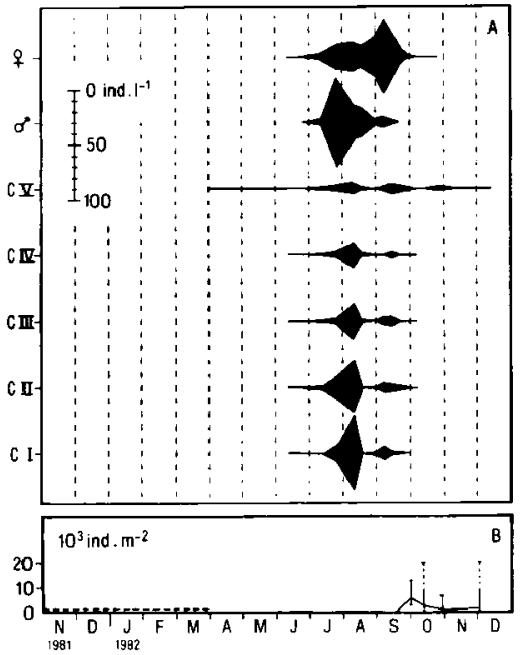

Fig. 6. Succession des stades copépodites et adultes pélagiques de Thermocyclops oithonoides (A) et évolution comparée des subadultes en diapause dans le sédiment (B). 


\subsection{4. - Thermocyclops crassus}

Th. crassus, espèce strictement estivale à Créteil, ne forme jamais une population très importante ( $\mathrm{fig}$. 3 et 7 ).

En 1982, la levée de diapause a été observée de mars à avril. Les copépodites IV ont alors poursuivi leur développement et les premières reproductions ont été observées en mai (fig. 7). Trois générations se sont succédé de juin à octobre, avec un maximum de 43 copépodites + adultes $I^{-1}$ ]e 11 août. La levée de diapause est totale : aucun copépodite IV n'a été trouvé dans le sédiment entre mai et septembre.

L'induction de la diapause se produit à la fin de l'été : 18000 ind. $\mathrm{m}^{-2}$ pendant l'automne $1981 \mathrm{et}$ 20500 ind. $\mathrm{m}^{-2}$ en 1982 à la même période, soit un potentiel de recolonisation du lac de 4 ind. $1^{-1}$ et 4,6 ind. $!^{-1}$ respectivement.

Dans les lacs européens, Th. crassus présente normalement une diapause hivernale (Smyly 1964 , Stella \& Margaritora, 1971 in Kiefer 1978 b, Amoros 1973). Au contraire, en région tropicale où elle est communément répandue, l'espèce est pérenne (Burgis 1971).
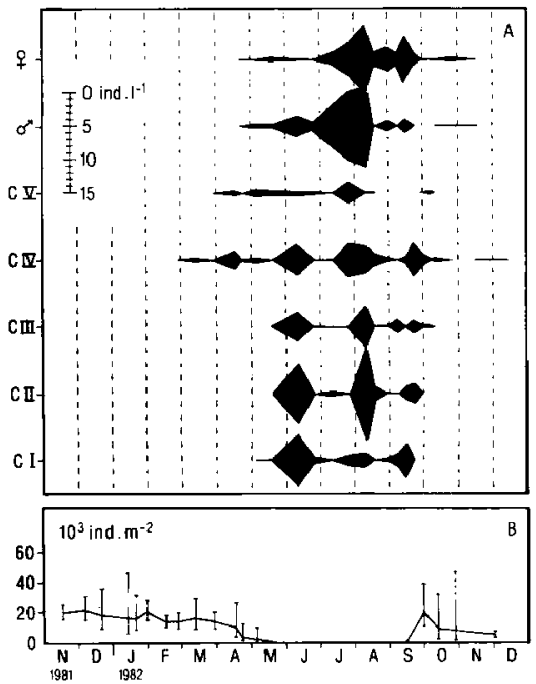

Fig. 7. Succession des stades copépodites et adultes pélagiques de Thermocyclops crassus (A) et évolution comparée des copépodites IV en diapause dans le sédiment (B).

\subsection{5. - Acanthocyclops robustus}

Dans le lac de Créteil, A. robustus est pérenne. Son développement est surtout estival et son abondance est maximale de juin à septembre. On observe généralement une période de reproduction automnale : il en a été ainsi en novembre 1981. Les individus de cette génération se métamorphosent lentement mais régulièrement pour atteindre leur maturité sexuelle en mars-avril ( $\mathrm{fig} .3$ et 8 ). Cette espèce présente dans d'autres lacs européens une diapause hivernale. Ainsi, dans le lac de Constance, des copépodites IV d'A. robustus apparaissent en zone pélagique en mars-avril, la population régressant en novembre (Einsle 1977). Une courte diapause hivernale existe dans le lac de Tjeukemeer; elle affecte les stades copépodites III à $\mathbf{V}$, en proportions variables selon les années (Vijverberg 1977).

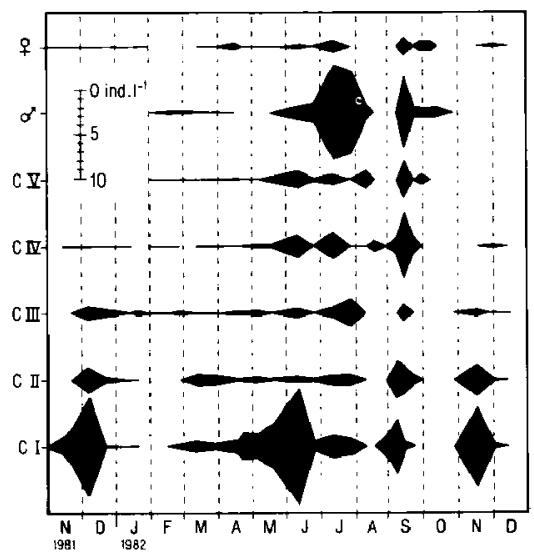

Fig. 8. Succession des stades copépodites et adultes péla giques d'Acanthocyclops robustus.

Les conditions climatiques moins rigoureuses de Créteil doivent expliquer l'absence de diapause d'A. robusius. Il faut noter cependant que, si l'examen des sédiments n'a pas permis de déceler une phase benthique, il existe néanmoins une incontestable accumulation d'A. robustus à proximité des sédiments pendant la saison froide : de novembre à avril, l'abondance près du substrat est 5,3 fois supérieure à celle de la zone pélagique. 


\section{4. - Discussion}

\section{1. - Induction de la diapause}

Le ròle essentiel de la photopériode dans l'induction de la diapause a été démontré expérimentalement sur C. vicinus (Einsle 1964, Spindler 1971) et sur deux espèces du genre Diacyclops (Watson \& Smallman 1971 a). Che\% ces dernières, une augmentation de la température provoque une diminution de la photopériode critique; en revanche l'effet de la photopériode sur l'induction de la diapause est identique à $16^{\circ} \mathrm{C}$ et $20^{\circ} \mathrm{C}$ chez C. vicinus (Spindler loc. cit.).

Dans le lac de Créteil, l'entrée en diapause des Cyclopides est observee pour une photopériode généralement inférieure à celle signalée pour d'autres lacs européens:

- C. vicinus: 14 h à Créteil (fin avril-début mai); $15 \mathrm{~h}$ dans le lac de Constance (Einsle, 1964); 15-17 h (juillet-août) dans le lac d'Eglwys Nynydd (George 1973) : 15 h $07 \mathrm{mn}$ - 16 h $24 \mathrm{mn}$ (mai-juin) dans le réservoir de Tjeukemeer (Vijverberg 1977).

- D. bicuspidalus : 12 h 30 mn à Créteil (mars) ; 14 h $13 \mathrm{mn}$ - 15 h $34 \mathrm{mn}$ (avril-mai) dans le réservoir de Tjeukemeer ( $\mathrm{Vij}$ verberg loc cit.).

- Th. oithonoides : 13 h $30 \mathrm{mn}-14$ hà Créteil (septembre) ; fin août dans le lac de Pääjärvi (Sarvala 1979).

- Th. crassus : 13 h $30 \mathrm{mn}-14$ h à Créteil (septembre); fin septembre-début octobre dans les étangs des Dombes, France (Amoros 1973).

Ces différences tiennent probablement au caractère plus septentrional, ou parfois plus continental, des autres milieux d'étude vis-à-vis du lac de Créteil. Ces résultats sont en accord avec ceux de Sarvala (loc. cit.) qui indique une augmentation de la photopériode critique avec la latitude, tant pour les espèces à diapause estivale que pour les espèces à diapause hivernale.

Pendant la saison froide, des subadultes de $T h$. oithonoides sont régulièrement observés en phase active dans l'eau libre du lac de Créteil. Cela traduit la possibilité d'un arrêt dẹ développement sans pénétration dans le sédiment (" diapause active " selon Elgmork 1980) chez quelques individus de cette espèce. Un tel phénomène a déjà été signalé chez
Mesocyclops leuckarti (Einsle 1968), Cyclops abyssonum (Einsle 1975), Cyclops scutifer (Halvorsen \& Elg. mork 1976). Sarvala (1979) a formulé l'hypothèse que, si la photopériode constituait le facteur essentiel provoquant l'arrêt de développement de $M$. leuckarti, d'autres facteurs et notamment la température interviendraient pour la pénétration des subadultes dans le sédiment. A Créteil, les conditions hivernales peu rigoureuses sont ainsi probablement à l'origine de la diapause active observée chez une fraction de la population de $T h$ oithonoides.

\section{2. - Levée de la diapause}

La température (Eigmork 1959, Smyly 1961, 1962, George 1973), la teneur en oxygène du milieu (Wierzbicka 1962, Szlauer 1963), les stimulus mécaniques (Elgmork 1959, Nilssen \& Elgmork 1977), constituent autant de facteurs immédiats susceptibles de provoquer ou d'accélérer l'émergence des individus diapausants.

A Créteil, les variations de la teneur en oxygène (fig. 1) sont difficiles à mettre en relation directe avec la réactivation des Cyclopides en diapause. Par ailleurs, Smyly (1962) sur M. leuckarti et George (1973) sur $C$. vicintus signalent des taux d'émergence identiques entre des échantillons aérés mécaniquement ou stagnants.

Le rôle de la température dans la réactivation des individus en diapause a été étudié expérimentalement chez M. leuckarti (Fryer \& Smyly 1954, Smyly 1962) et C. vicinus (George 1973). Chez les deux espèces le taux d'émergence est relié à la température : la durée de la période de latence de $M$. leuckarti est d'autant plus longue que la température est basse et la sensibilité des individus à une hausse de température augmente avec le temps passé en diapause : le taux d'émergence de $C$, vicinus augmente de même avec la température, mais une période préalable d'exposition au froid est nécessaire pour provoquer une réactivation massive des individus. Cependant, le retour à la vie active a finalement lieu chez les deux espèces quelle que soit la température.

Dans le lac de Créteil, les premières émergences de $C$. vicinus sont observées en fin d'été mais, en accord avec les résultats expérimentaux de George (loc. cit.). la réactivation massive des copepodites a lieu en janvier et février après que la température de l'eau ait atteint sa valeur minimale avec $2^{\circ} \mathrm{C}$. De 
même, l'apparition régulière de Th. oithonoides et de Th. crassus au cours des mois de mars ęt avril est vraisemblablement liée à l'augmentation de la température observée au cours de cette même époque.

Watson \& Smallman (1971 a) ont distingué dans la diapause des Cyclopides une « phase réfractaire " caractérisée par une absence de réactivité aux stimulus de l'environnement et une phase " compétente " pendant laquelle la sensibilité des individus aux facteurs du milieu s'accroit. Il semble qu'à Créteil la temperature constitue le facteur essentiel de synchronisation des émergences pendant cette période de diminution de l'intensité de la dormance.

\section{3. - Signification adaptative de la diapause}

La diapause hivernale de $T h$. oithonoides et de $T h$. crassus permet à ces formes thermophiles de se maintenir dans un environnement climatique défavorable. L'évolution latitudinale du cyle de vie de ces espèces montre du nord au sud une diminution progressive de l'intensité de la diapause qui peut aboutir à une forme de vie totalement pérenne. Ainsi, des subadultes de Th. oithonoides présentent une diapause active à Créteil et $T h$. crassus, en diapause vraie à Créteil, est perenne dans les régions tropicales (Burgis 1971). De mème, A. robustus observé en diapause hivernale dans le réservoir de Tjeukemeer (Vijverberg 1977) et le lac de Constance (Einsle 1977), a un développement continu dans le lac de Créteil.

Cet aspect de défense vis-à-vis des conditions climatiques parait moins essentiel pour $C$. vicinus. Dans le lac de Créteil, une population pélagique peut se maintenir au cours de certains étés et assurer sa reproduction. La prédation exercée par les poissons planctonophages - particulièrement la prédation sélective exercée par les alevins sur les plus grands individus planctoniques - constitue probablement le facteur essentiel de limitation du développement estival de cette espèce (Lescher-Moutoué 1984, Lescher-Moutoué \& al., 1985).

La période d'induction de la diapause de $C$. vicinus à Créteil précède les dates d'apparaition des alevins de Percidés et de gardons et la diapause cons. titue de ce fait un refuge efficace à la prédation. Il semble ainsi logique de considérer la pression de prédation des alevins comme une force sélective pro- bable vis-à-vis de la diapause de $C$. vicinus, comme cela a été suggéré par Nilssen (1977) et Elgmork \& al. (1978) pour d'autres espèces du genre Cyclops.

La diminution de la photopériode critique observée $d u$ nord au sud, tant pour les espèces à diapause estivale que pour les espèces à diapause hivernale, constitue un ajustement des cycles saisonniers des Cyclopides aux variations latitudinales des contraintes abiotiques mais aussi biotiques du milieu.

Enfin, Gamier \& Lescher-Moutoué (1984), étudiant les successions saisonnières des populations planctoniques du lac de Créteil, ont observé que les principaux groupes zooplanctoniques, mais aussi les principales espèces, ont des maximums souvent décalés, ce qui pourrail traduire un évitement de la compétition alimentaire des individus vis-à-vis du phytoplancton. Bien qu'à notre connaissance l'importance de la compétition en tant que force sélective de la diapause n'ait pas été démontrée, il n'en est pas moins vrai que la dormance dans le sédiment contribue au partage des ressources par la ségrégation temporelle de certaines espèces.

Travaux eltés

Amoros (C.). 1973. - Evolution des populations de Cladocères et de Copépodes dans trois étangs piscicoles de la Dombes. Annls Limnol, 9 (2) : 135-155.

Birge (E.A.) \& Juday (C.). 1908. - A summer resting stage in the development of Cyclops bicuspidatus Trans. Wiscons. Acad. Sci., Arts Lett., 16: 1-9.

Burgis (M.J.). 1971. - The ecology and production of Copepods, particularly Thermocyclops hyalinus, in the tropical Lake George. Uganda. Freshwat. Biot., 1 : 169.192.

Cardinal (C.). 1981. - Composition, evolution et structure des populations phycoplanctoniques du lac de Créteil (Val-de-Marne) abordées sous differents aspects. These de $3 *$ cycle, Univ. Paris VI, $128 \mathrm{p}$.

Cardinal (C.) \& Garnier (J.). 1980. - Structure, évolution et production du phytoplancton du lac de Créteil. Actes $25^{\circ}$ Congrès nat. A.F.L., Paris : 147-156.

Champeau (A.). 1963. - Note préliminaire sur divers stades de résistance chez les Copépodes dans les eaux temporaires de la Camargue. Rapports et PV réunions CIESMM, 17 (3): 805-810.

Champeau (A.). 1970. - Recherches sur l'écologie et l'adaptation à la vie latente des Copépodes des eaux temporaires provençales et corses. Thèse Fac. Sc. Aix-Marseille, 360 p.

Cole (G.A.). 1953, - Notes on Copepod encystment. Ecology. 34 : 208-211.

Einsle (U.). 1964. - Die Gattung Cyclops in Bodensee. Arch. Hydrobiol., $60: 133-199$.

Einsle (U.). 1965. - Okologische Studien an einer pelagisch lebender Population von Diacyclops bicuspidatus (Crust. Cop). Gewässer und Abweasser, $39 / 40: 102.117$.

Einsle (U.). 1968. - Die Gatiung Mesocyclops im Bodensee. Arch. Hydrobial, 64 : 131-169. 
Einsle (U.). 1975. - Revision der Gattung Cyclops s. str. speziel der abyssorum Gruppe. Mem. Ist. Ihal. Idrobiol., 32 : 57-219.

Einsle (U.). 1977. - Untersuchungen zum Auftreten von Acamthocyclops robusrus (Crust. Cop.) im Bodensee-Oberse. Arch. Hydrobiol. $79: 382-396$

Elgmork (K,), 1958. - On the phenology of Mesocyclops oithonoi des (G.O. Sars). Verh. Intemat. Verein. Limnol. 13: 778-784.

Elgmork (K.). 1959. - Seasonal cccurence of Cyclops strentus strenuus in relation to environment in small water bodies in southern Norway. Folia Limnol. Scand, $11: 196 \mathrm{p}$.

Elgmork (K.). 1962. - A bottom resting stage in the planktonic freshwater Copepod Cyclops scutifer Sars. Oikos, 13 (2): 306-310.

Elgmork (K.). 1967. - Ecological aspects of diapause in copepods. Proc. Symp. Crustacea, 3 : $947-954$.

Elgmork (K). 1973. - Bot tom resting stages of planktonic cyclopoid copepods in meromictic Lakes. Verh. Intemat. Verein. Limnol., 18 : 1474-1478.

Elgmork (K.). 1980. - Evolutionary aspects of diapause in freshwater Copepods. In C. Kerfoot (ed,), Evolution and Ecology of Zooplankton Communities. Spec. Symp., University Press of New England (London), $3: 411-417$.

Elgmork (K.) \& Nilssen (J.P.). 1978. - Equivalence of copepod and insect diapause. Verh. Internat. Verein. Limmol, $20: 2511-2517$.

Elgmork (K.), Nilssen (J.P.), Broch (T.) \& Ovrevik (R.). 1978. - Life cycle strategies in neighbouring populations of the copepod Cyclops scutifer Sars. Verh. Intemat. Verein. Limmol, 20 : 2518.2523

Elliott (J.M.). 1971. - Sorne methods for the statistical analysis of samples of benthic invertebrates. Freshwater Biol. Ass. Sci. Publ, 25, 144 p.

Fryer (G.) \& Smyly (W.J.P.). 1954. - Some remarks on the resting stages of some freshwater cyclopoid and harpacticoid copepods. Ann. Mag. Nat. Hist., $7:$ 65-72.

Garnier (J.). 1982. - Production primaire d'une sablière (lac de Créteil, Val-de-Marne). Etude de certains facteurs de contróle. Thèse 3e cycle, Univ. Paris VI, 12t p.

Garnier (J.). \& Lescher-Moutoué (F.). 1984. - Successions saisonnières phyto-zooplanctoniques dans un écosystème lacustre peu profond (lac de Créteil, France). Verh. Intemar. Verein. Liminol., 22 (sous presse).

Garreau (F.). 1978. - Dynamique des populations de Rotifères planctoniques du lac de Créteil. Thèse 3e cycle, Univ. Paris VI, $144 \mathrm{p}$.

George (D.G.). 1973. - Diapause in Cyclops vicinus. Oikos, 24 : 136.142

Gerdeaux (D.). 1980. - Les Poissons. In Faune et flore du Lac de Créteil (Val-de-Marne). Chalut. Publ. Lab. Zool., E.N.S., Paris, 17 : ISBN 2-72880063-4; 63-66.

Halvorsen (G.) \& Elgmork (K). 1976. - Vertical dist ribution and seasonal cycle of Cyclops scutifer Sars (Crustacea, Copepoda) in two oligotrophic lakes in southern Norway. Norw. J. Zool., 24 : 143.160 .

Kiefer (F.) 1963. - Bemerkenswerte Copepodenfunde in Pelagial des Bodensees. Schweiz-Zeitschr. Hydrol., $25 ; 30-39$.

Kiefer (F,) 1978 a. - Zur Kennt nis des Diacyclops thomasi (S.A. Forbes, 1882) (Copepoda Cyclopoida). Crustaceana, 34 : 214-216.

Kiefer (F.). 1978 b. - Das Zooplankton der Binnengewässer. 2 Teil. Freilebende Copepoda. E. Schweizerbartsche Verlags., Stut1gart, $343 \mathrm{p}$.

Lescher-Moutoué $(F)$. 1984. - Structure, biomasse et production des Copépodes Calanoides d'un écosystème lacustre peu profond (lac de Creteil, France). Acta Oecologica, Oecol. Gener, 5 , 2. 91-108.
Lescher-Moutoué (F.) \& Bertrand (1.Y.). 1980. - Variations saisonnières des Crustacés planctoniques du lac de Créteil (France). C.R. 25" congrès A.F.L., Parts, 157.169.

Lescher-Moutoué (F.), Gamier (J.) \& Pourriot (R.). (1985)-Interac. lions entre les peuplements planctoniques el piscicole du lac de Créteil : impact d'une reproduction exceptionnelle de Percidés. Bull. Ecol. (sous presse).

Milbrink (G.). 1971. - A simplified tube bottom sampler. Oikos, $22: 260.263$.

Nilssen (J.P.). 1977. - Cryptic predation and the demographic strategy of two limnetic cyclopoid copepods. Mem. Ist. Ital. Idrobiol., 34 : 187.196.

Nilssen (J.P.). 1978. - On the evolution of life histories of limnetic cyclopoid copepods. Mem. Ist. Mal. Idrobiol., 36 : 193.214.

Nilssen (J.P.). 1980. - When and how to reproduce: A dilemma for limnetic Cyclopoid Copepods. In W.C. Kerfoot (ed.), Evolution and Ecology of Zooplankton Communities. Spec. Symp., Uni. versity Press of New England (London), 3 ; 418-426.

Nils sen (J.P.) \& Elgmork (K.). 1977. Cyclops abyssorum - Life cycle dynamics and habital selection. Mem. Ist. Ital. Idrobiol., 34 : 197.238.

Sarvala (J.). 1979. - Benthic resting periods of pelagic Cyclopoids in an oligotrophic lake. Holarct. Ecol., 2 : 88.100.

Smyly (W.J.P.). 1961. - The life-cycle of the freshwater copepod Cyclops leuckarti Claus in Esthwaite water. J. Anim. Ecol., 30 : 153.169.

Smyly (W.J.P.). 1962. - Laboratory experiments with stage V copepodids of the freshwater copepod. Cyclops leuckarti Claus, from Windermere and Esthwaite water. Crustaceana, 4: 273.280 .

Smyly (W.J.P.). 1964. - An investigation of some benthic Entomostraca of three lakes in northern italy. Mem. Ist. Ital. Idrobiol., $17: 33-56$.

Spindler (K.D.). 1971. - Dormanzaus lösung und dormanzcharakteristika beim süßwassercopepoden Cyclops vicinus. Ecol. Jb. Physiol., 76 : 139.151.

Szlauer (L.). 1963. - The resting stages of Cyclopidae in Stary Dwor Lake. Pol. Arch. Hydrobiol., $10: 385.394$.

Testard (P.). 1983. - Le lac de Créteil (région parisienme) ; un lac de sablière peu profond. In Problèrnes d'écologie : st ructure et fonctionnement des écosystèmes limniques. M. Lamotte et F. Bourlière Eds. Masson Paris: 113-159.

Vijverberg (J.). 1977. - Population structure, life histories and abundance of Copepods in Tieukemeer, the Netherlands. Freshwat. Biol., $7: 579-597$.

Watson (N.H.F.) et Smallman (B.N.). 1971 a. - The role of photo period and temperature in the induction and ternination of an arrested development in two species of freshwater cyclopid copepods. Can. J. Zool, $49: \mathbf{8 5 5 . 8 6 2}$.

Watson (N.H.F.) et Smallman (B.N.) 1971 b. - The physiology of diapause in Diacyclops navus Herrick (Crustacea, Copepoda). Can. J. Zool., 49 : $1449-1454$.

Wierzbicka (M.). 1962. - On the resting stage and mode of life of some species of Cyclopoida, Pol Arch. Hydrobiol, $10: 215-229$.

Wierzbicka (M.). 1966. - Les résultats des recherches concernant l'etal de repos (resting stage) des Cvclopoida. Verh. Internat. Verein. Limnol., 16 : 592.599.

Wierzbicka (M.). 1972. - The metabolic products of copepodites of various Cyclopoida species during their resting stage. Pol. Arch. Hydrobiol., 19 (3) : 279.290. 